\title{
RECUPERAÇÃO DE PARTÍCULAS GROSSAS DE QUARTZO
}

\author{
M. A. SEGER ${ }^{*}$, R. T. RODRIGUES \\ Universidade Federal do Rio Grande do Sul \\ marco.seger@ufrgs.br*
}

Artigo submetido 03/04/2019 e aceito em 09/08/2019

DOI: 10.15628/holos.2019.8475

\section{RESUMO}

A influência do tamanho de partícula tem sido foco de décadas de estudos na flotação de minérios, aumentar o limite superior do tamanho de partícula na flotação ainda é um grande desafio para indústria do processamento mineral. Um dos benefícios da flotação de partículas grossas, esta relacionado com a diminuição dos custos de cominuição, bem como a redução da geração de partículas finas e ultrafinas. 0 processo de floto-elutriação tem se mostrado uma alternativa promissora para a recuperação de partículas grossas (+ $250 \mu \mathrm{m})$. A floto-elutriação, além de operar com menores velocidades superficiais de gás, o que reduz significativamente a turbulência dentro da zona de coleta, diminuindo assim o "detachment", a floto-elutriação trabalha com um fluxo contracorrente (água de elutriação), o que
\end{abstract}

diminui a velocidade de sedimentação das partículas, aumentando assim o tempo de residência, e a probabilidade de colisão partícula/bolha. O objetivo do presente trabalho foi estudar a influência da velocidade superficial do gás (Jg) e do líquido (JI) na recuperação de partículas grossas $(-500+250 \mu \mathrm{m})$ por floto-elutriação e comparar com a flotação mecânica (célula tipo Denver). Os resultados mostraram que o aumento na velocidade superficial do líquido (de 0,63 $\mathrm{cm} . \mathrm{s}^{-1}$ para $1,05 \mathrm{~cm} . \mathrm{s}^{-1}$ ), produziu um ganho de recuperação por floto-elutriação (5,94\% para $24,10 \%)$. A velocidade superficial do gás foi o parâmetro com menor influência na recuperação. Em comparação com a célula mecânica, a floto-elutriação apresentou um ganho de $14 \%$ na recuperação

PALAVRAS-CHAVE: Floto-elutriação, elutriação, partículas grossas, flotação.

\section{RECOVERY OF COARSE PARTICLES QUARTZ}

\begin{abstract}
The influence of particle size has been the focus of decades of ore flotation studies, increasing the upper limit of particle size in flotation is still a major challenge for the mineral processing industry. One of the benefits of coarse particle flotation is related to lower comminution costs as well as reduced generation of fine and ultra fine particles. The floto-elutriation process has shown to be a promising alternative for coarse particle recovery $(+250 \mu \mathrm{m})$. Floto-elutriation, in addition to operating at slower gas velocities, which significantly reduces turbulence within the collection zone, thus decreasing detachment, floto-elutriation works with a countercurrent flow (elutriation water). which decreases
\end{abstract}

the settling velocity of the particles, thereby increasing the residence time, and the likelihood of particle / bubble collision. The objective of the present work was to study the influence of gas $(\mathrm{Jg})$ and liquid $(\mathrm{Jl})$ surface velocity on coarse particle recovery $(-500+250 \mu \mathrm{m})$ by flotationelutriation and to compare it with mechanical flotation (Denver cell). The results showed that the increase in liquid surface velocity (from $0.63 \mathrm{~cm} . \mathrm{s}^{-1}$ to $1.05 \mathrm{~cm} . \mathrm{s}^{-1}$ ) produced a recovery gain by floto-elutriation $(5.94 \%$ to $24.10 \%)$. The surface velocity of the gas was the parameter with the least influence on the recovery. Compared to the mechanical cell, floto-elutriation showed a $14 \%$ recovery gain.

KEYWORDS: Floto-elutriation, elutriation, coarse particles, flotation. 


\section{INTRODUÇÃO}

O processo de flotação de minérios possui alta eficiência de separação em um intervalo de tamanho de partícula relativamente restrito, aproximadamente 20-150 $\mu \mathrm{m}$ (Gaudin, Schuhmann \& Schlechten, 1942; Jameson, Nguyen \& Ata, 2007). A importância do tamanho da partícula nas etapas de colisão, adesão e transporte tem sido amplamente investigada (Glembotskii, Klassen \& Plaksin, 1972; Trahar, 1981; Bazin \& Proulx, 2001).

A baixa recuperação de partículas grossas por flotação é um dos principais problemas da indústria mineral. A diminuição na recuperação de partículas grossas está principalmente relacionada a problemas de adesão bolha-partícula (detachment), enquanto que para partículas finas, o problema deve-se a baixa probabilidade de colisão (Dai, Fornasiero \& Ralston, 2000; Gontijo, Fornasiero \& Ralston, 2007; Awatey, Thanasekaran, Kohmuench, Skinner \& Zanin, 2013; 2014). Estudos realizados por Gontijo et al, (2007) e Awatey et al, (2014) apresentaram elevadas recuperações para partículas com tamanhos de até $1180 \mu \mathrm{m}$, para altos valores de ângulo de contato.

A baixa recuperação de partículas grossas ocorre principalmente devido a: (1). elevada massa; (2). Baixo grau de liberação e disseminação entre espécies minerais; (3). Grau de adsorção do coletor (baixa hidrofobicidade); (4). Estabilidade de espuma; (5). Poder de levitação das bolhas na célula; e (6). Tempo de residência (Ata \& Jameson, 2013; Dunne, 2011; Feng \& Aldrich, 1999; Mazon, 2006; Moudgil \& Gupta, 1989; Oliveira \& Aquino, 2005; Schubert \& Bischofberger 1979).

A floto-elutriação, ao contrário de um separador de leito fluidizado tradicional, opera com injeção contínua de bolhas de ar, mantendo as condições de fluxo não turbulento e, assim, obtendo maior seletividade na separação por flotação de partículas hidrofóbicas. Em comparação com a flotação convencional, a floto-elutriação opera com menores velocidades superficiais de gás, resultando em uma menor turbulência e, portanto, uma condição hidrodinâmica mais favorável à flotação de partículas grossas. Também, o contínuo transbordo do material elutriado aumenta a recuperação dos agregados bolha-partícula (Awatey et al, 2014; Kohmuench et al, 2007; 2010; 2013; Wingate \& Kohmuench, 2016).

\section{EXPERIMENTAL}

\subsection{Materiais e métodos}

\subsubsection{Amostra de Quartzo}

Os estudos foram realizados com uma amostra de areia quartzosa, com composição química 89.6\% $\left(\mathrm{SiO}_{2}\right), 5.08 \%\left(\mathrm{Al}_{2} \mathrm{O}_{3}\right), 5.32 \%$ de outros minerais, com densidade 2.63 g.cm $\mathrm{cm}^{-3}$ (picnometria). $\mathrm{O}$ material foi peneirado a seco nas malhas: $35 \#(500 \mu \mathrm{m})$ e $60 \#(250 \mu \mathrm{m})$. O produto $(-500+250 \mu \mathrm{m})$ foi homogeneizado em uma lona plástica com 100 tombos e quarteado pelo método de fracionamento de pilha cônica. As alíquotas obtidas foram armazenadas em baldes de plástico. 


\subsubsection{Estudos de elutriação no hidrofloat ${ }^{\mathrm{TM}}-$ E.}

Para avaliar a recuperação por elutriação foram realizados experimentos sem a adição de coletor. $600 \mathrm{~mL}$ de polpa com $20 \%$ de sólidos (foi utilizado $20 \%$ de sólidos por questões operacionais de recirculação do underflow) foi alimentada ao HydroFloat ${ }^{\text {tm }}$ por gravidade, o qual foi previamente preenchido com uma solução de $\left(9 \mathrm{mg} \cdot \mathrm{L}^{-1}\right)$ espumante. $\mathrm{O}$ espumante utilizado foi o flotanol D 25 (metil éter de polipropilenoglicol - Clariant $^{\odot}$ ). Autores relatam que acima dessa concentração de coalescência ( $9 \mathrm{mg} \cdot \mathrm{L}^{-1}$ ), a bolha deixa de coalescer e alcança seu tamanho médio final de Sauter (Cho \& Laskowski, 2002; Grau \& Heiskanen, 2005). O fluxo ascendente de água para elutriação foi mantido através da injeção de uma solução de flotanol D 25 (9 mg. $\left.\mathrm{L}^{-1}\right)$. O fluxo de ar foi controlado por rotâmetro e a pressão por um filtro regulador $\left(2 \mathrm{Kgf.cm}{ }^{-2}\right)$. $O$ tempo inicial (TO) dos experimentos foi contado a partir do início do transbordamento da espuma. O tempo de duração dos experimentos foi de 10 minutos. O fluxo de polpa descarregado no fundo da célula foi mantido constante por uma bomba, que recircula o underflow para a alimentação. Esta condição de recirculação (Figura 1) foi adotada para se obter um tempo de retenção mais longo e um efeito scavenger. No final de cada ensaio, o concentrado e o rejeito foram, filtrados, secos e pesados. Foi utilizada água da rede de abastecimento público de Porto Alegre-RS (DMAE). Todos os ensaios foram realizados a temperatura ambiente $\left(\sim 23^{\circ} \mathrm{C}\right)$

\subsubsection{Estudos de floto-elutriação no HidroFloat ${ }^{\mathrm{TM}}-F E$}

Para avaliar a floto-elutriação o procedimento foi o mesmo utilizado nos estudos de elutriação, entretanto a polpa foi condicionada com o coletor Alkyl Eter Amina (flotigan EDA-Clariant (C) na concentração de $180 \mathrm{gt}^{-1}$ durante 5 minutos e depois alimentada (transferida por gravidade) para o HydroFloat ${ }^{\mathrm{T} M}$. Em ambos os casos, as velocidades superficiais do gás $(0,2 ; 0,27 ; 0,33$ e 0,39 $\left.\mathrm{cm} . \mathrm{s}^{-1}\right)$ e as velocidades do líquido $\left(0,63 ; 0,84 \mathrm{e} 1,05 \mathrm{~cm} \cdot \mathrm{s}^{-1}\right)$ foram variadas. Os experimentos foram realizados em duplicatas

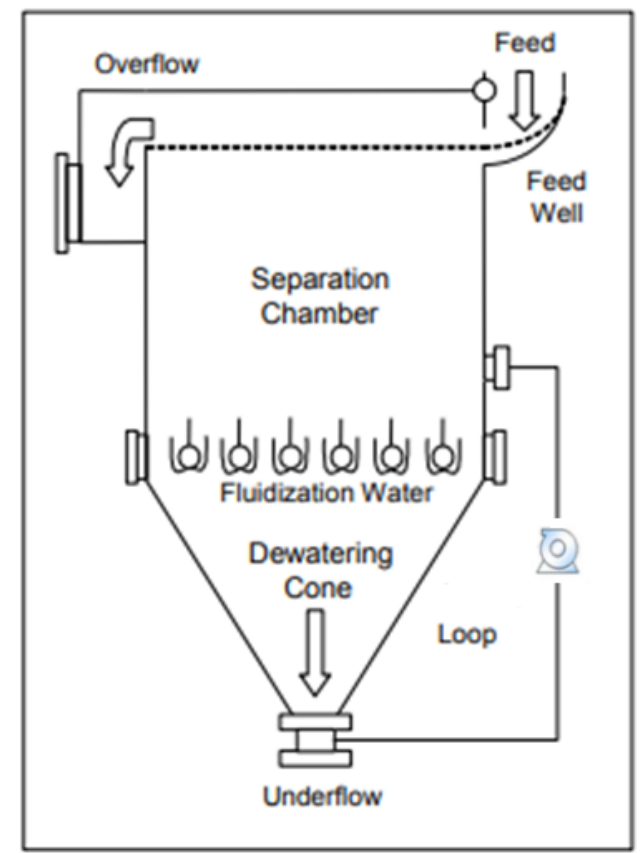

Figura 1: Esquema ilustrativo do sistema utilizado. 
A flotação real $\left(\mathrm{F}_{\mathrm{t}}\right)$ foi calculada com a Equação 1.

$F_{t}=F_{e}-E$

onde: $F_{e}=$ recuperação na floto-elutriação e $E$ = recuperação na elutriação.

\subsubsection{Estudos de flotação convencional- FC}

Os experimentos foram realizados em uma célula mecânica de laboratório (Denver ${ }^{\circledR}$ ) com capacidade de 1,5 L. A polpa contendo $9 \%$ de sólidos (em peso) e pH 7 foi condicionada sob agitação (800 rpm - $5 \mathrm{~min}$ ) com o coletor Alkyl Eter Amina (flotigan EDA-Clariant ${ }^{\circ}$ ) na concentração de 180 $\mathrm{gt}^{-1}$ e $9 \mathrm{mg} \cdot \mathrm{L}^{-1}$ de flotanol D 25. A vazão de ar utilizada na flotação variou entre 4 e 5,5 min. $\mathrm{L}^{-1}$. O tempo de flotação foi de 10 minutos. Todos os experimentos foram realizados em duplicata. No final de cada ensaio, o concentrado e o rejeito foram, filtrados, secos e pesados. Foi utilizada água da rede de abastecimento público de Porto Alegre-RS (DMAE). Todos os ensaios foram realizados a temperatura ambiente $\left(\sim 23^{\circ} \mathrm{C}\right)$

\section{RESULTADOS E DISCUSSÃO}

3.1. Elutriação e floto-elutriação

A Tabela 1 mostra as recuperações médias obtidas para todas as condições testadas.

Tabela 1: Condições experimentais e recuperações.

\begin{tabular}{c|ccc|c}
\hline$J_{\mathrm{I}}\left(\mathbf{c m} \cdot \mathbf{s}^{-\mathbf{1}}\right)$ & $\mathrm{J}_{\mathrm{g}}\left(\mathbf{c m} \cdot \mathbf{s}^{-\mathbf{1}}\right)$ & $\begin{array}{c}\text { Recuperação } \\
\text { Elutriação }\end{array}$ & $\begin{array}{c}\text { Recuperação } \\
\text { Floto-elutriação }\end{array}$ & $\begin{array}{c}\text { Recuperação } \\
\text { Flotação real }\end{array}$ \\
\hline 0,63 & $\mathbf{( \% )}$ & $\mathbf{( \% )}$ & $\mathbf{( \% )}$ \\
0,63 & 0,20 & 3,17 & 5,94 & 2,77 \\
0,63 & 0,33 & 4,55 & 8,51 & 3,96 \\
0,63 & 0,39 & 4,82 & 7,98 & 3,16 \\
0,84 & 0,20 & 11,81 & 5,94 & 1,43 \\
0,84 & 0,27 & 11,31 & 17,23 & 5,42 \\
0,84 & 0,33 & 10,37 & 16,76 & 5,45 \\
0,84 & 0,39 & 9,05 & 15,06 & 4,69 \\
1,05 & 0,20 & 15,71 & 17,87 & 8,82 \\
1,05 & 0,27 & 18,45 & 24,10 & 8,39 \\
1,05 & 0,33 & 17,73 & 20,04 & 1,59 \\
& & & 19,66 & 1,93
\end{tabular}




\begin{tabular}{l|lll|l}
1,05 & 0,39 & 14,78 & 19,01 & 4,23 \\
\hline
\end{tabular}

Pode-se observar que a recuperação na floto-elutriação (com coletor) foi sempre superior a recuperação na elutriação (sem coletor). A diferença entre a recuperação com e sem coletor (Ft) variou durante os testes, mostrando que existe um ponto ótimo de operação.

\subsection{Efeito da velocidade superficial do liquido.}

A Figura 2 mostra os resultados da variação da velocidade da superfícial do líquido na recuperação por elutriação e floto-elutriação. A velocidade superficial de gás foi mantida constante $\left(0,2 \mathrm{~cm} \cdot \mathrm{s}^{-1}\right)$. Com o aumento da velocidade superficial do liquido, observou-se um ganho de recuperação tanto com o coletor (5,94\% para $24,10 \%)$ como sem o coletor $(3,17 \%$ para $14,78 \%)$. Este aumento na recuperação foi devido ao efeito conjugado do arraste + flotação real.

O que determinará se as partículas ascenderão ou sedimentarão será a diferença entre a velocidade de sedimentação da partícula, e a velocidade superficial do líquido. Se a velocidade de sedimentação for maior, as partículas tenderão a sedimentar e serão direcionadas para a corrente de underflow; caso contrário, as partículas ascenderão e serão coletadas na corrente do overflow (Honaker \& Mondial, 1999). Com a adição da água de elutriação, há uma redução na velocidade de sedimentação das partículas, em virtude disso, o tempo de residência das partículas na célula de flotação, a probabilidade de colisão partícula/bolha e a probabilidade de flotação, tenderão a aumentar. 


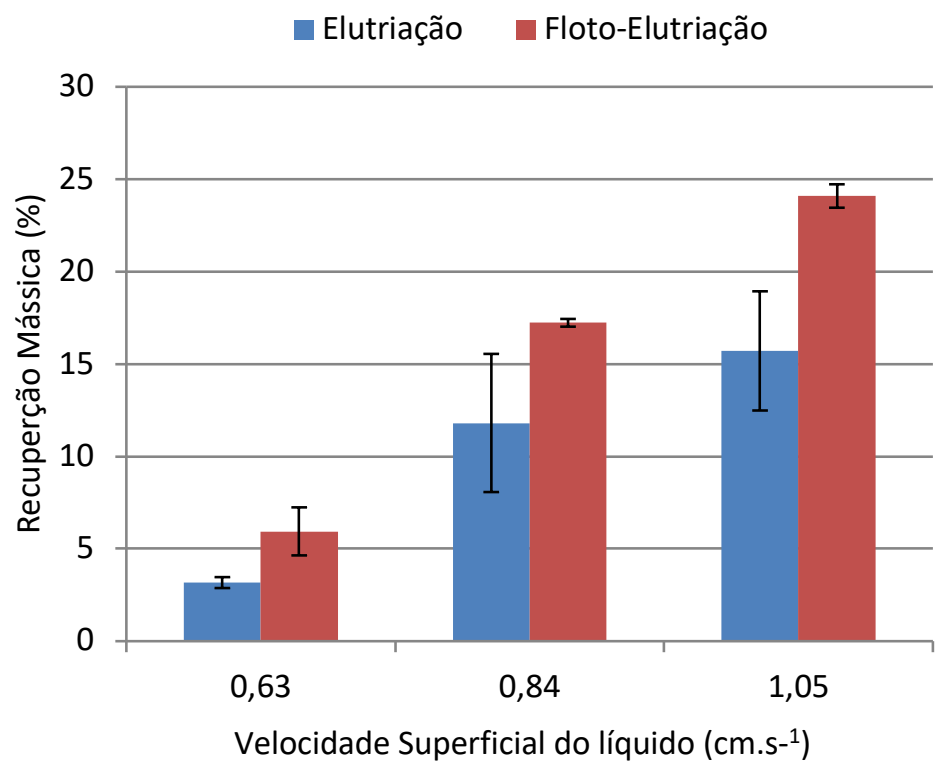

Figura 2: Recuperação por elutriação e floto-elutriação em função da velocidade superficial do líquido, quando o $\mathrm{Jg}=0.2\left(\mathrm{~cm} \cdot \mathrm{s}^{-1}\right)$.

Para se obter uma recuperação máxima, a velocidade superficial da água deve ser aumentada para um ponto ótimo, a fim de evitar a sedimentação das partículas (Awatey et al, 2013). Também, segundo Brum (2004), com o emprego da agua de elutriação, a uma redução nos valores do holdup. Se por um lado a presença ou o execesso de sólidos resultam no aumento do tempo de retenção das bolhas de ar na zona de coleção, o fluxo ascendente de água de elutriação ajuda no transporte destas e consequentemente reduz o conteúdo de ar nessa região da coluna.

\subsection{Efeito da velocidade superficial de gás.}

A figura 3 apresenta as recuperações em função da variação da velocidade superficial de gás. Verificou-se que a variação da velocidade superficial de gás $\left(0,2 \mathrm{~cm} \cdot \mathrm{s}^{-1}\right.$ a $\left.0,39 \mathrm{~cm} \cdot \mathrm{s}^{-1}\right)$, não causou impacto significativo na recuperação.

A maior recuperação mássica foi de $24,1 \%$ obtida através da floto-elutriação para $J_{\mathrm{g}}=0,2$ $\mathrm{cm} \cdot \mathrm{s}^{-1} \mathrm{e} \mathrm{J}_{I}=1,05 \mathrm{~cm} \cdot \mathrm{s}^{-1}$, mas o maior aumento obtido com a adição do coletor foi na condição $\mathrm{J}_{\mathrm{g}}=$ $0,39 \mathrm{~cm} \cdot \mathrm{s}^{-1} \mathrm{e} \mathrm{J}=0,84 \mathrm{~cm} \cdot \mathrm{s}^{-1}$, onde apresentou um ganho de mais de $94 \%$ de recuperação em relação à elutriação. Este ponto deve ser o de maior seletividade, uma vez que a diferença entre o processo de elutriação e a floto-elutriação foi maior (8,82\%), indicando um melhor desempenho da flotação real. 


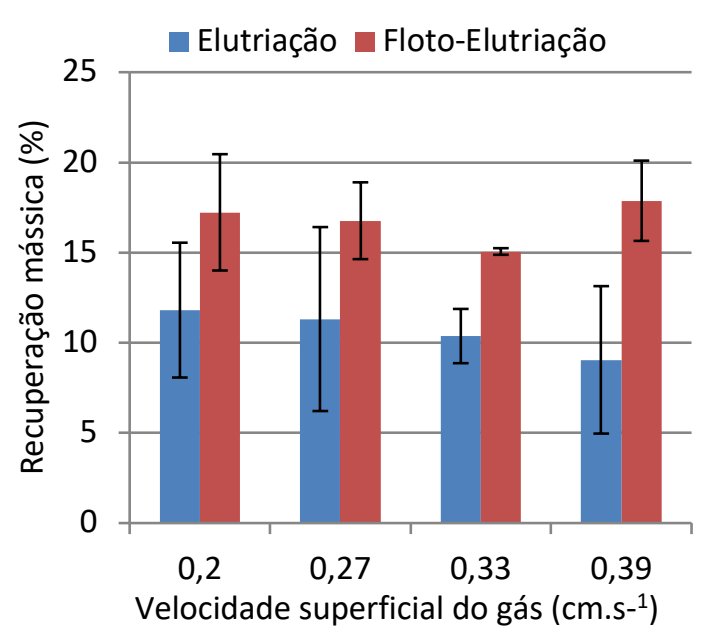

(a)

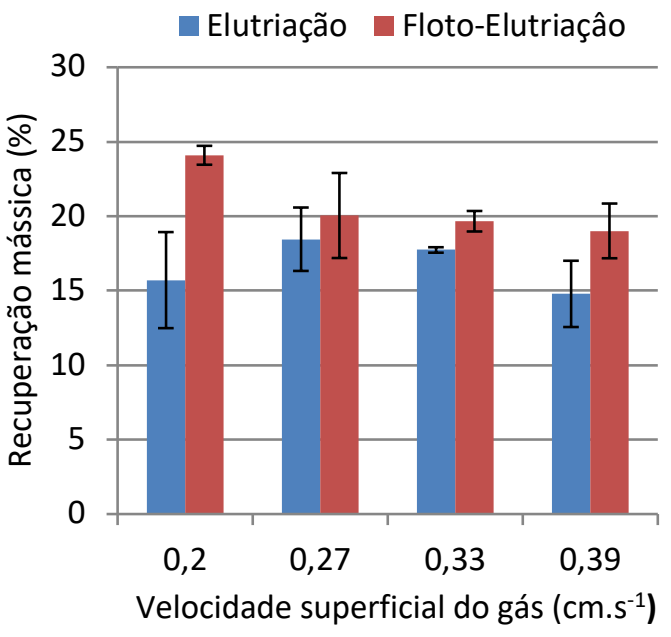

(b)

Figura 3: Recuperação por elutriação e floto-elutriação em função da velocidade superficial do gás, quando o Jl é: (a) $0.84\left(\mathrm{~cm}^{-1} \mathrm{~s}^{-1}\right)$ (b) $1.05\left(\mathrm{~cm} . \mathrm{s}^{-1}\right)$.

Diversos autores relatam que dentro dos limites de estabilidade da coluna, a recuperação do mineral flotado é normalmente crescente com o aumento da vazão de gás até atingir um máximo. Este crescimento na recuperação deve-se ao aumento da área superficial de bolhas introduzidas na coluna. Porém, esse aumento na recuperação não foi observado, talvez, o acréscimo da vazão de gás pode ter alterado as condições hidrodinâmicas para um regime mais turbulento, prejudicando o processo.

\subsection{Flotação convencional (célula denver)- FC}

Foram realizados estudos de flotação convencional a fim de avaliar a recuperação mássica total e comparar com os resultados obtidos nos estudos de floto-elutriação.

A figura 4 apresenta a recuperação mássica em função da variação da velocidade superficial de gás, pode-se notar que com a variação da velocidade superficial de gás houve um acréscimo na recuperação.

A máxima recuperação obtida nos experimentos com célula de flotação mecânica foi de $10,5 \%$ para um $\mathrm{Jg}=0,52 \mathrm{~cm} \cdot \mathrm{s}^{-1}$. Esta baixa recuperação de partículas grossas de quartzo foi observada por Vieira e Peres (2007), e foi associada ao tipo de coletor, pH e ao potencial-zeta. 


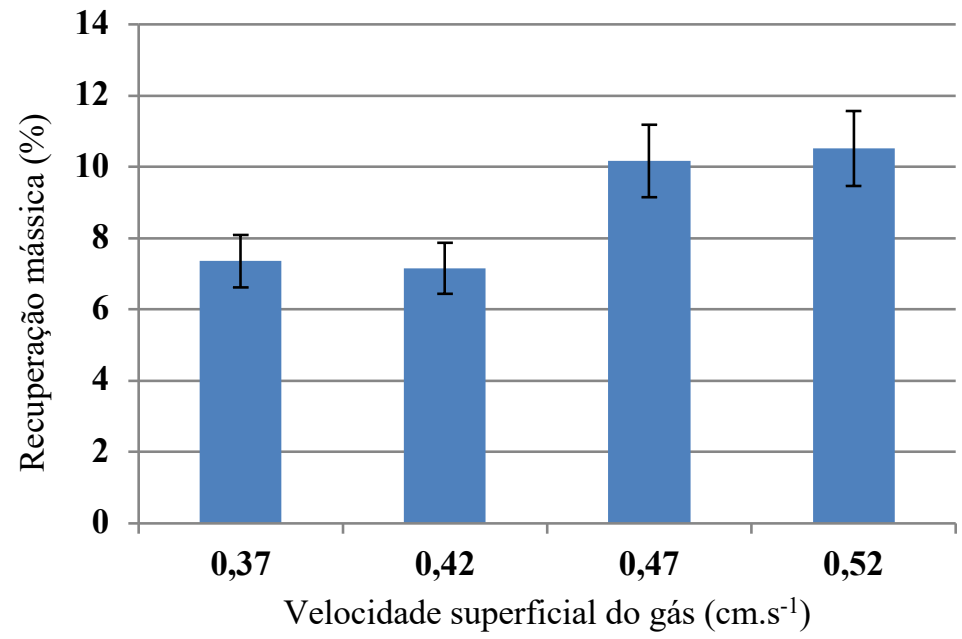

Figura 4: Recuperação mássica por flotação convencional (FC), em função da variação da velocidade superficial do gás.

A figura 5 apresenta a máxima recuperação obtida por floto-elutriação e por flotação convencional, pode ser perceber que os resultados obtidos por floto-eluriação (24.10\%) foram muito superiores aos de flotação convencional (10.5\%).

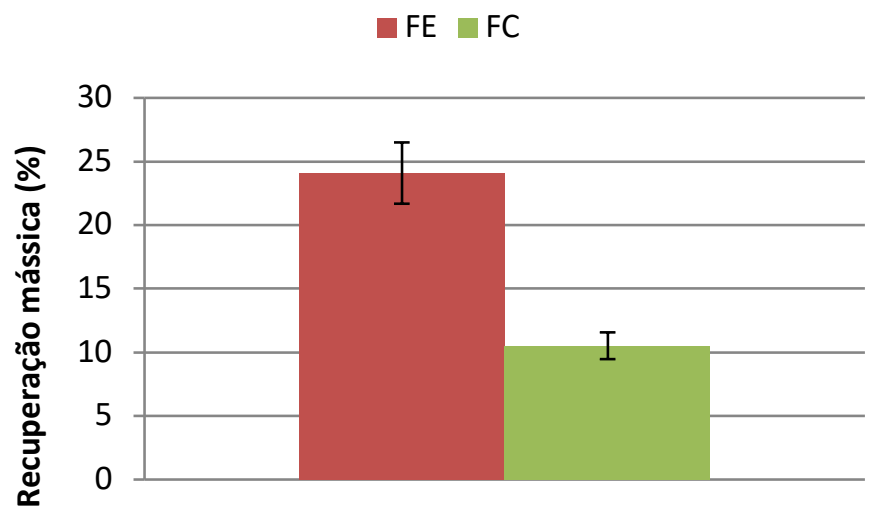

Figura 5: Máxima recuperação obtida por Floto-elutriação (FE) e flotação convencional (FC).

De acordo com Awatey et al (2013), geralmente, a recuperação de partículas grossas é baixa em células do tipo Denver por duas razões principais; a elevada turbulência criada dentro da célula pelo "impeller" e a presença da camada de espuma torna difícil para que as partículas grossas sejam transferidas da polpa para a espuma. Ao contrário, a floto-elutriação opera sob condições quiescentes que criam um ótimo ambiente hidrodinâmico para a eficiente flotação de partículas grossas. 


\section{CONCLUSÃO}

Foram avaliados dois parâmetros na recuperação de partículas grossas por floto-elutriação, a velocidade superficial do gás e do liquido. A variação da velocidade superficial do gás, não apresentou um impacto significativo na recuperação, porem pode-se notar uma variação na recuperação por flotação real $(1,43 \%$ a $8,82 \%)$, indicando a existência de um ponto de maior eficiência no processo.

As recuperações mássicas na FE em relação à $\mathrm{FC}$ foram maiores, devido ao transporte de massa via adesão bolhas/partículas ser assistido pela ação de elutriação (fluxo ascendente de água). Houve um maior grau de arraste de partículas, que é independente das espécies mineralógicas (não seletivo) e depende da distribuição de tamanhos e densidade das partículas minerais.

Em comparação ao método de flotação convencional, o HidroFloat ${ }^{\mathrm{TM}}$ se mostrou uma alternativa viável para a recuperação de partículas grossas, pois houve um aumento de $13,6 \%$ na recuperação (10,5\% na flotação em célula mecânica e $24,1 \%$ via floto-eleutriação).

\section{BIBLIOGRAFIA}

1. Ata, S., \& Jameson, G.J. (2013). Recovery of coarse particles in the froth phase - A case study. Minerals Engineering, v. 45, p. 121-127.

2. Awatey, B.; Thanasekaran, H.; Kohmuench, J.N.; Skinner, W. \& Zanin, M. (2013). Optimization of operating parameters for coarse sphalerite flotation in the hydrofloat fluidised-bed separator. Minerals Engineering, v. 50-51, p. 99-105.

3. Awatey, B.; Thanasekaran, H.; Kohmuench, J.N.; Skinner, W. \& Zanin, M. (2014). Critical contact angle for coarse sphalerite flotation in a fluidised-bed separator vs. A mechanically agitated cell. Minerals Engineering, v. 60, p. 51-59.

4. Bazin, C., \& Proulx, M. (2001). Distribuition of reagentes down a flotation bank to improve the recovery of coarse particles. International Journal of Mineral Processing, v. 61, n. 1, p. 1- 12.

5. Brum, I.A.S. (2004). Concentração de partículas minerais grossas de fluorita em coluna de flotação. 120 p. Tese (Doutorado em engenharia) - PPGE3M, UFRGS, Porto Alegre, 2004.

6. Cho, Y.S., \& Laskowski, J.S. (2002). Effect of flotation frothers on bubble size and foam stability, Int. J. Miner. Process. 64 69-80. doi:10.1016/S0301-7516(01)00064-3.

7. Dai, Z.; Fornasiero, D. \& Ralston, J. (1999). Particle-bubble attachment in mineral flotation. Journal of Colloid and Interface Science, v. 217, n. 1, p. 70-76.

8. Dunne, R.C. (2012). The journey of the coarse particle through the pulp and froth in flotation. In: International Mineral Processing Congress, 26. Nova Deli. Proceedings...v. 1, p. 1259-1268.

9. Feng, D., \& Aldrich, C. (1999). Effect of particle size on flotation performance of complex sulphide ores. Minerals Engineering, v. 12, n. 7, p. 721-731.

10. Gaudin, A.M.; Schuhmann, R. \& Schlechten, A.W. (1942). The effect of size on the behavior of galena particles-Flotation Kinetics II. J. Phys. Chem., v. 64, p. 902-910.

11. Glembotskii, V.A., Klassen, V.I. \& Plaksin, I.N., (1972). The effect of mineral particle size on flotation. In: Flotation, p. 230-250 (Chapter 2). 
12. Gontijo, C.F.; Fornasiero, D. \& Ralston, J. (2007). The limits of fine and coarse particle flotation. The Canadian Journal of Chemical Engineering, v. 85, n. 5, p. 739-747.

13. Grau, R.A., \& Heiskanen, K. (2005). Bubble size distribution in laboratory scale flotation cells, Miner. Eng. 18 1164-1172. doi:10.1016/j.mineng.2005.06.011.

14. Honaker, R. Q., \& Mondial, K. (1999). Dynamic Modeling of a Fine Coal Separations in a Hindered-bed Classifier. Coal Preparation, v. 21, p 211-232.

15. Jameson, G.J.; Nguyen, A.V. \& Ata, S. (2007). The flotation of fine and coarse particles, Froth Flotat. Century Innov. p. 339-372.

16. Kohmuench, J.; Thanasekaran, H. \& Seaman, B. (2013). Advances in Coarse Particle FlotationCopper and Gold. In: metplant Conference, Perth. Proceedings...p. 378-386.

17. Kohmuench, J.N.; Mankosa, M.J.; Kennedy, D.G.; Yasalonis, J.L.; Taylor, G.B. \& Luttrell, G.H. (2007). Implementation of the hydrofloat Technology at the South Fort Meade Mine, Minerals and Metallurgical Processing, v. 24, n. 4, p. 264-270.

18. Kohmuench, J.N.; Mankosa, M.J.; Yan, E.S.; Wyslouzil, H. \& Christodoulou, L. (2010). Advances in Coarse Particle Flotation Industrial Minerals. In: International Mineral Processing Congress, 25., Brisbane. Proceedings... p. 2065- 2076.

19. Mazon, R. (2006). Efeito do tempo de residência sobre a recuperação metálica da flotação colunas reversa de minério de ferro. 112 p. Dissertação (Mestrado em engenharia) PPGEM, UFOP, Ouro Preto, 2006.

20. Moudgil, B. M., \& Gupta, D. (1989). Flotation of coarse phosphate particles. Advances in coal and mineral processing using flotation, p. 164-168

21. Oliveira, M. L., \& Aquino, J. A. (2005). Aspectos relevantes das colunas de flotação. In: Encontro Nacional de Tratamento de Minérios e Metalurgia Extrativa, 21., Natal. Anais... p. 44- 52.

22. Schubert, H., \& Bischofberger, C. (1979). On the optimization of hydrodynamics in flotation processes. In: International Mineral Processing Congress, 13., Varsóvia. Proceedings... Part B, v. 2b, p. 1261-1287.

23. Trahar, W. J. (1981). A rational interpretation of role particle size in flotation. International Journal of Mineral Processing, v. 2, p. 289-327.

24. Vieira, A.M., \& Peres, A.E.C. (2007). The effect of amine type, $\mathrm{pH}$, and size range in the flotation of quartz, Miner. Eng. 20. 1008-1013. doi:10.1016/j.mineng.2007.03.013

25. Wingate, E., \& Kohmuench, J. (2016). An optimized approach to phosphate recovery. Beneficiation of Phosphates: Comprehensive Extraction, Technology Innovations, Advanced Reagents, p. 43. 